\title{
Games in Tetrads: Segregation, Recombination, and Meiotic Drive
}

\section{Citation}

Haig, David Addison. 2010. Games in tetrads: Segregation, recombination, and meiotic drive. The American Naturalist 176(4): 404-413.

\section{Published Version}

doi:10.1086/656265

\section{Permanent link}

http://nrs.harvard.edu/urn-3:HUL.InstRepos:8944745

\section{Terms of Use}

This article was downloaded from Harvard University's DASH repository, and is made available under the terms and conditions applicable to Other Posted Material, as set forth at http:// nrs.harvard.edu/urn-3:HUL.InstRepos:dash.current.terms-of-use\#LAA

\section{Share Your Story}

The Harvard community has made this article openly available.

Please share how this access benefits you. Submit a story.

Accessibility 


\title{
Games in Tetrads: Segregation, Recombination, and Meiotic Drive
}

\author{
David Haig \\ Department of Organismic and Evolutionary Biology, Harvard University, Cambridge, Massachusetts 02138 \\ Submitted March 6, 2010; Accepted June 11, 2010; Electronically published August 20, 2010
}

\begin{abstract}
AвSTRACт: The two alleles at a heterozygous locus segregate during meiosis, sometimes at meiosis I and sometimes at meiosis II. The timing of segregation is determined by the pattern of crossing-over between a locus and its attached centromeres. Genes near centromeres can exploit this process by driving against spores from which the genes separated at meiosis I. Other genes, located distal to centromeres, can benefit from driving against spores from which they separated at meiosis II. Asymmetric female meiosis is particularly susceptible to such forms of drive. Selection on modifiers of recombination favors changes in the location of chiasmata that increase the proportion of tetrads of high average fitness by changing the timing of segregation. Such changes increase the frequency of driving alleles. This source of selection on recombination does not depend on effects on linkage disequilibrium. Recombinational responses to meiotic drive may contribute to sex differences in overall recombination and sex differences in the localization of chiasmata.
\end{abstract}

Keywords: meiosis, meiotic drive, polar body, recombination, centromere, segregation.

\section{Introduction}

Dissolutions of partnerships (matrimonial and otherwise) are often times of conflict as former collaborators fall out over who gets what in the division of joint property. Meiosis similarly marks the parting of ways of temporary allelic partners who no longer have shared goals but may now compete for limited resources or reproductive opportunities. Separation of alleles (segregation) may occur at either the first or the second meiotic division, depending on the pattern of crossing-over (recombination) between a locus and its centromere. Two-step meiosis alleviates some of the risks associated with segregation by creating uncertainty about when alleles part company (Haig and Grafen 1991; Haig 1993a), but despite this feature, the division of meiotic spoils is not always equitable (Lyttle 1991; Burt and Trivers 2006).

* E-mail: dhaig@oeb.harvard.edu.

Am. Nat. 2010. Vol. 176, pp. 404-413. (C) 2010 by The University of Chicago. 0003-0147/2010/17604-51976\$15.00. All rights reserved.

DOI: $10.1086 / 656265$
Meiosis in embryophytes (land plants) occurs in diploid sporocytes to produce tetrads of four haploid spores. After the completion of meiosis, spores are released from their tetrad into the locule of a diploid sporangium, where spores undergo rapid expansion and accumulation of nutrients (McCormick 2004). There are two principal reasons why a spore might benefit from imposing a cost on another spore within its tetrad. First, if a spore's expected fitness increases with the amount of its nutrient reserves, then the members of a tetrad compete for parental resources. A spore that took more than its fair share would enhance its own fitness at a cost to other spores that received less as a result. Second, if the members of a tetrad are dispersed and germinate together, then spores within a tetrad may compete after dispersal for limited resources, space, or reproductive opportunities. In this case, a spore might benefit from preemptively disabling (or otherwise compromising) its potential competitors before dispersal. Although this article focuses on meiosis in embryophytes, its models have a wider relevance to meiosis in other organisms.

\section{Games within Tetrads}

Interactions within meiotic tetrads can be viewed as an evolutionary game played between the two alleles of a sporocyte. Tetrads will be named after the genotype of the sporocyte from which the tetrad is derived. Thus, a $D d$ tetrad contains two spores carrying a $D$ allele and two spores carrying a $d$ allele, whereas a $D D$ tetrad contains two spores carrying an egg-derived (madumnal) $D$ allele and two spores carrying a sperm-derived (padumnal) $D$ allele. Madumnal and padumnal are used solely as labels to identify the two allelic positions and do not denote any difference in role, strategy, or payoffs. The fitness payoffs for madumnal alleles will be given in matrices of the form

$$
\left[\begin{array}{cc}
0 & w_{D d} \\
w_{d D} & -1
\end{array}\right],
$$

where $w_{d d}=0$ is the null payoff for $d$ in a $d d$ tetrad, $w_{D d}$ 


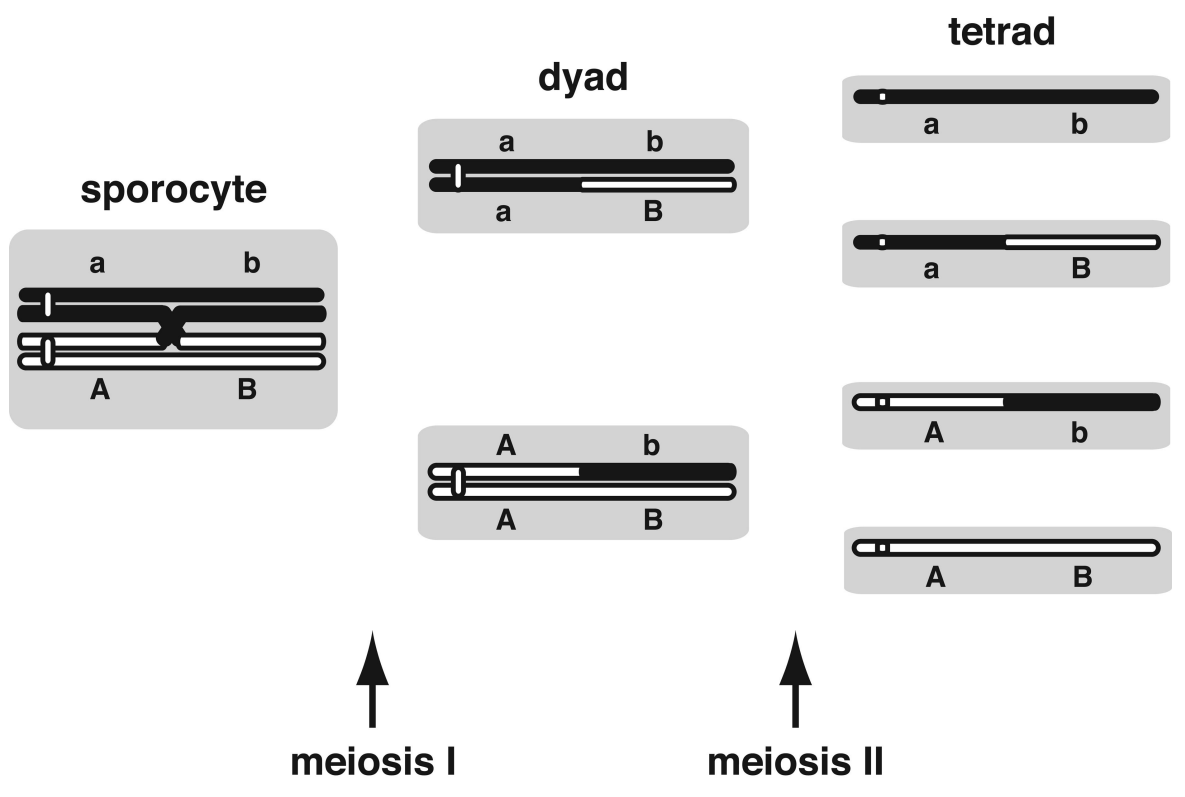

Figure 1: Meiotic segregation of a bivalent with one crossover. Homologous centromeres segregate at meiosis I, then sister centromeres separate at meiosis II. At loci proximal to the crossover $(A a)$, loss of heterozygosity (segregation) occurs at the first division. At loci distal to the crossover $(B b)$, segregation occurs at the second division.

is the payoff to $D$ in a $D d$ tetrad, $w_{d D}$ is the payoff to $d$ in a $D d$ tetrad, and $w_{D D}=-1$ is the payoff to $D$ in a $D D$ tetrad. The definition $w_{D D}=-1$ means that the driving allele $D$ is associated with a fitness cost when homozygous and that payoffs are proportional to the difference in fitness between spores from the two homozygous tetrads $\left(w_{d d}-w_{D D}=1\right)$.

If gametes unite at random, then $D$ can invade a population fixed for $d$ if $w_{D d}>0$ and $d$ can invade a population fixed for $D$ if $w_{d D}>w_{D D}$. This article will be concerned with situations of drive, defined as $w_{D d}>0>w_{d D}$, and, in particular, with cases where drive reduces average fitness within $D d$ tetrads, $w_{D d}+w_{d D}<0$. The matrix describes a prisoner's dilemma if $w_{D d}>0$ and $w_{d D}<w_{D D}$, in which case, $d$ will be eliminated at equilibrium. The matrix describes a game of chicken if $w_{D d}>0$ and $w_{d D}>w_{D D}$, in which case, both alleles will be maintained in the population at a polymorphic equilibrium.

\section{Relatedness within Tetrads}

Spores whose nuclei separated at meiosis II will be called sisters, whereas spores whose nuclei separated at meiosis I will be called cousins (Haig 1993a). Thus, each spore in a tetrad has one sister and two cousins. Before meiosis, each allele at a heterozygous locus is present in two copies. The two copies either part company at meiosis I (first- division segregation) or remain together at meiosis I but separate at meiosis II (second-division segregation). In the former case, sister spores will carry different alleles. In the latter case, sister spores will carry the same allele (fig. 1).

The probability that two spores share the same allele at a heterozygous locus will be called the relatedness of the spores at that locus. Relatedness of sisters $\left(r_{\mathrm{s}}\right)$ and cousins $\left(r_{\mathrm{c}}\right)$ depends on the probability of second-division segregation $(\Phi): r_{\mathrm{s}}=1-\Phi$ and $r_{\mathrm{c}}=\Phi / 2$. Thus, relatedness differs for different loci on a single chromosome. In theory, a sister could be $r_{\mathrm{s}}=0$ for a gene at one locus, $r_{\mathrm{s}}=1$ for a gene at another locus, and all values between. The corresponding range of relatedness for a cousin is $r_{\mathrm{c}}=0$ to $r_{\mathrm{c}}=1 / 2$ (Haig 1986).

The probability of second-division segregation $(\Phi)$ at a locus is determined by the number of crossovers $(n)$ between the locus and its centromere:

$$
\Phi=\sum_{n=0} \frac{2}{3}\left[1-(-0.5)^{n}\right] p_{n}
$$

where $p_{n}$ is the probability of $n$ crossovers (Mather 1935; equation assumes no chromatid interference; fig. 2). As a rough general statement, $r_{\mathrm{s}}$ is high (and $r_{\mathrm{c}}$ correspondingly low) near centromeres, with $r_{\mathrm{s}}$ and $r_{\mathrm{c}}$ converging on onethird at loci farther along chromosome arms. Precise statements, however, depend on the probability distribution of 


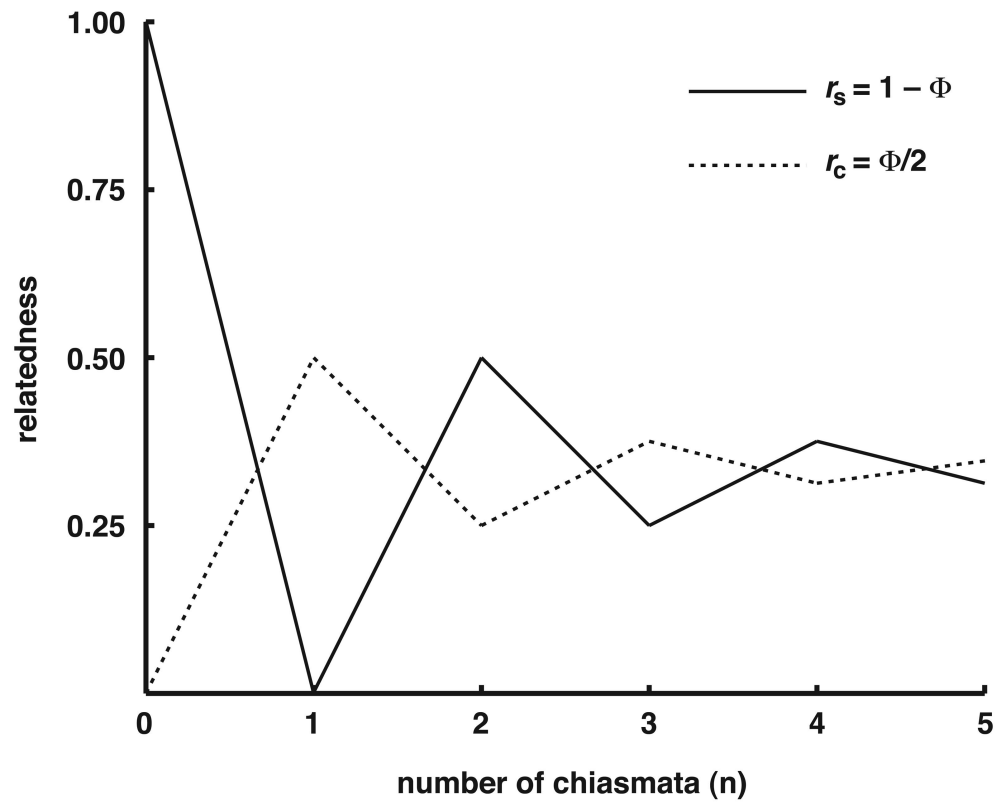

Figure 2: Probability that two spores share the same allele because of identity by recent common descent ("relatedness") as a function of the number of chiasmata between a locus and its centromere. Spores that are descended from the same dyad cell are sisters. Spores that are descended from different dyad cells are cousins. Relatedness to sisters $\left(r_{\mathrm{s}}\right)$ and to cousins $\left(r_{\mathrm{c}}\right)$ depends on the probability of second-division segregation $(\Phi)$.

$p_{n}$. For example, $\Phi=2 / 3\left(r_{\mathrm{s}}=r_{\mathrm{c}}=1 / 3\right)$ for all $x$ in the interval $(0,1)$ when $p_{0}=(1-x) / 3, p_{1}=(2-x) / 3$, and $p_{2}=2 x / 3$. This example shows that equal relatedness to cousins and sisters is theoretically possible at all points in a chromosome interval that does not include the centromere. Each chromosome has at least one locus, its centromere, for which $\Phi=0\left(p_{0}=1\right)$ but need not have any loci for which $\Phi=1$, because the latter requires an obligate single crossover between locus and centromere $\left(p_{1}=1\right)$.

\section{Hide-and-Seek}

Predictable patterns of relatedness within tetrads create opportunities for $D$ alleles that target spores with a low probability of carrying $D$. This section begins with some simple games in which spores interact in pairs. Matched pairs carry the same allele. Mismatched pairs carry different alleles. A $D d$ tetrad contains two $D$ spores and two $d$ spores that can be grouped as two matched pairs ( $D$ paired with $D$ and $d$ paired with $d$ ) or two mismatched pairs (each $D$ paired with $d$ ). When pairs are matched, $d$ spores receive the null payoff and $D$ spores receive -1 . When pairs are mismatched, $d$ spores receive $-t$ and $D$ spores receive $s(s, t>0)$.

If cousins or sisters are paired, then the proportion of matched and mismatched pairs produced by $D d$ sporocytes is determined by $\Phi$. Thus, the number of chiasmata between the $D$ locus and its centromere affects fitness within tetrads and will, for this reason, be subject to natural selection. The average payoff for a spore from a $D d$ tetrad is $(s-t) / 2$ in mismatched pairs but $-1 / 2$ in matched pairs. Therefore, selection on unlinked modifiers favors mismatched pairs when $s-t>-1$ but matched pairs when $s-t<-1$. For simplicity of presentation, I ascribe differential effects on sisters and cousins to pairing of spores, but physical pairing is not necessary for genes to have differential effects on the two classes of relatives.

\section{Exploit-a-Sister}

Suppose that each spore interacts with its sister. Firstdivision segregation of a $D d$ sporocyte results in matched pairs: $D$ spores receive -1 and $d$ spores receive the null payoff. An unlinked modifier has expected payoff $-1 / 2$. Second-division segregation results in mismatched pairs: $D$ spores receive $s$ and $d$ spores receive $-t$. An unlinked modifier has expected payoff $(s-t) / 2$. Payoffs at the $D$ locus are

$$
\left[\begin{array}{cc}
0 & s \Phi-(1-\Phi) \\
-t \Phi & -1
\end{array}\right]
$$


$D$ can invade a population fixed for $d$ when $\Phi>1 /(1+$ $s)$. $d$ can invade a population fixed for $D$ when $\Phi<1 / t$.

Stable polymorphic equilibria exist when $1 / t>\Phi>$ $1 /(1+s)$. Inspection of these inequalities reveals that a necessary condition for a stable polymorphism is $s-t>$ -1 (mismatch advantage). At such an equilibrium, unlinked modifiers obtain a higher expected payoff from $D d$ tetrads when segregation occurs at meiosis II (mismatched sisters) than when it occurs at meiosis I (matched sisters). Therefore, selection on modifiers of recombination favors increases in $\Phi$. I focus on drive systems that maintain polymorphic equilibria because these exert sustained selection on recombination, whereas drive systems that sweep to fixation exert only transient selection on recombination.

Whether increases in $\Phi$ are achieved by decreases or increases of crossing-over depends on the probability distribution of $p_{n}$. For example, if $p_{0}=1-x$ and $p_{1}=x$, then selection on unlinked modifiers favors increases in $x$. If, however, $p_{1}=1-y$ and $p_{2}=y$, then selection favors decreases in $y$. In general, selection for increased $\Phi$ favors shifts from even to odd numbers of crossovers (fig. 2 ). Increases in $\Phi$ are associated with higher equilibrium frequency of $D$, with $D$ fixed whenever $\Phi>1 / t$ (possible only if $t>1$ ).

\section{Exploit-a-Cousin}

Suppose that each spore interacts with a cousin, such that the tetrad is organized as two pairs of cousins. Mismatched pairs are formed in all cases of first-division segregation and half the cases of second-division segregation in $D d$ tetrads, with combined probability $\Psi=1-\Phi / 2$. Therefore, exploit-a-cousin has the same payoffs as exploit-asister, with $\Psi$ substituted for $\Phi$. Polymorphic equilibria occur when $1 / t>\Psi>1 /(1+s)$ and require mismatch advantage, $s-t>-1$.

Selection on unlinked modifiers favors increases of $\Psi$, corresponding to decreases of $\Phi$. Therefore, selection favors shifts from odd to even numbers of crossovers, opposite of exploit-a-sister. Suppose that there is initially exactly one crossover between the $D$ locus and its centromere $\left(\Phi=1, p_{1}=1\right)$. A modifier could reduce $\Phi$ in two ways. First, the obligate crossover could be suppressed, or shifted to the other side of $D$, with probability $x\left(p_{0}=\right.$ $\left.x, p_{1}=1-x\right)$. The least value of $\Phi$ achievable by this route is $\Phi=0$ when $p_{0}=1$. $D$ would become fixed in the population once $\Phi<1 /(s+1)$. Alternatively, a second crossover could be inserted between $D$ and its centromere with probability $y\left(p_{1}=1-y, p_{2}=y\right)$. The least value of $\Phi$ achievable by this route is $\Phi=1 / 2$ when $p_{2}=1$. Although the equilibrium frequency of $D$ also increases by this route, a polymorphic equilibrium is maintained at $\Phi=1 / 2$ if $s>1$.

The existence of polymorphic equilibria in exploit-asister and exploit-a-cousin requires that average fitness in heterozygous tetrads is higher in the presence of drive (spores interacting in mismatched pairs) than in the absence of drive (spores interacting in matched pairs). When there is a balanced polymorphism, selection favors modifiers that reduce $r_{\mathrm{s}}$ in exploit-a-sister but reduce $r_{\mathrm{c}}$ in exploit-a-cousin. Both forms of modification accentuate the existing system of drive but make less likely the opposite form of drive (because $r_{\mathrm{s}}+2 r_{\mathrm{c}}=1$ ). Such modifiers may increase or decrease crossing-over, depending on chromosome location.

\section{Random Pairing}

$D$ 's other copy is more likely to be located in the sister than in a randomly chosen cousin when $\Phi<2 / 3\left(r_{\mathrm{s}}>r_{\mathrm{c}}\right)$ but the reverse when $\Phi>2 / 3\left(r_{\mathrm{c}}>r_{\mathrm{s}}\right)$. Therefore, higher returns can be obtained by driving against a cousin rather than a sister when $\Phi<2 / 3$ and the reverse when $\Phi>$ $2 / 3$. When $\Phi=\Psi=2 / 3$, the payoffs of exploit-a-sister and exploit-a-cousin are identical, and $D$ can do no better than target a random spore within its tetrad. Haig (1993a) implied that $d$ is safest when $\Phi=0.5$ because this maximizes uncertainty about whether $d$ is present in a sister spore, but the arguments presented here suggest instead that $d$ is safest when $\Phi=2 / 3$ because this maximizes uncertainty about the location of $d$.

\section{Safety in Numbers}

Average relatedness within a tetrad is one-third, but relatedness to a spore from another tetrad in the same sporangium is one-half, for all loci, regardless of a gene's chromosomal location. Thus, spores of low relatedness may be partially hidden among spores of higher relatedness once spores from different tetrads become mixed together after their release into the locule of a sporangium.

Suppose that $n$ sporocytes contribute $4 n$ spores to the local neighborhood within a locule. Each spore has $4 n-1$ neighbors, of which $2 n-1$ carry a copy of the same allele as the focal spore. The average relatedness within the neighborhood is $r_{n}=(2 n-1) /(4 n-1)$, which approximates one-half for large $n$. Consider a game played within a sporangium in which spores are paired at random: $D$ spores in matched pairs each receive $-1, d$ spores in matched pairs each receive the null payoff, and $D$ receives $s$ and $d$ receives $-t$ in mismatched pairs. If $n$ is large, the expected payoffs are 


$$
\left[\begin{array}{cc}
0 & (s-1) / 2 \\
-t / 2 & -1
\end{array}\right]
$$

The magnitude of this "hiding effect" is reflected in the conditions for $D$ to invade a population fixed for $d$. If $D$ is paired with a random spore in its own tetrad, $w_{D d}>0$ for all $s>1 / 2$. However, if $D$ is paired with a random spore from another tetrad, $w_{D d}>0$ for the more stringent condition $s>1$.

\section{Winner Takes All}

All spores in a tetrad initially have unit fitness. One position in a tetrad is privileged such that a $D$ allele in this position causes the abortion of the other spores in its tetrad. The privileged $D$ spore has fitness $1+S$ and its cousins and sister have zero fitness. Analysis is trivial. $D$ will invade a population fixed for $d$ and spread to fixation if $S>1$, but mean fitness is reduced if $S<3$.

\section{Classical Drive}

Most previous discussion of meiotic drive has considered systems in which $D$ targets $d$ directly, independent of a spore's position within a tetrad. This can be approximated by a simple game in which $D$ and $d$ receive $s$ and $-t$ in Dd tetrads, namely

$$
\left[\begin{array}{cc}
0 & s \\
-t & -1
\end{array}\right]
$$

$D$ displaces $d$ from the population when $s>0$ and $t>1$. $D$ and $d$ are maintained at a polymorphic equilibrium when $s>0$ and $t<1$. The direct targeting of spores carrying $d$ can be viewed as a spiteful greenbeard acting in the small local population of the tetrad (Haig 1997; Gardner and West 2010). Exploit-a-sister (eq. [3]) reduces to classical drive (eq. [5]) when $\Phi=1$, because sisterhood is then a perfect marker of zero relatedness. Exploit-acousin reduces to classical drive when $\Phi=0$, because cousinhood is then a perfect marker of zero relatedness.

Representation of classical drive as an interaction between alleles at a single locus is a simplification. Rather, classical drive involves Killer alleles at a distorter locus disabling spores with Sensitive alleles at a linked responder locus. Thus, Killer at the first locus creates a selective advantage for spores with Resistant alleles at the second locus. Killer increases in frequency by genetic hitchhiking because it occurs on the same haplotype as Resistant (Lyttle 1991).

Haig and Grafen (1991) showed that selection on unlinked modifiers favors increased recombination between distorter and responder loci. At polymorphic equilibria, homozygosity for the Killer/Resistant haplotype is associ- ated with individual fitness costs that balance its segregation advantage in heterozygotes. Genes at loci that are unlinked to the haplotype gain no segregation advantage but experience the individual fitness cost. Therefore, natural selection on unlinked modifiers favors increased recombination between Killer and Resistant because decoupling drive increases a modifier's chances of being present in individuals of high fitness in subsequent generations. Natural selection, however, also favors inversions that prevent crossing-over between Killer and Resistant alleles (Charlesworth and Hartl 1978). The haplotype then behaves as a single locus. The process can be viewed as a race between selection on unlinked modifiers to increase recombination and eliminate drive and the occurrence of inversions that fix the driving haplotype.

\section{Asymmetries of Meiosis}

Pseudohomothallic ascomycetes include species in which nuclei that separated at meiosis I are paired within a common spore wall and species in which nuclei that separated at meiosis II are paired. Asci of Neurospora tetrasperma contain four binucleate ascospores (eight nuclei in total because of a postmeiotic mitosis). The two nuclei within each ascospore are daughters of meiotic cousins and therefore differ at loci that underwent first-division segregation. By contrast, ascospores of Podospora anserina contain two nuclei that are daughters of meiotic sisters and therefore differ at loci that underwent second-division segregation (Raju and Perkins 1994). Turner and Perkins (1991) have suggested that this pairing of nuclei may have evolved to neutralize certain forms of meiotic drive. These examples illustrate the possibility of arbitrary pairings of spores within tetrads.

Two principal kinds of cell division are recognized in plant sporogenesis (Furness et al. 2002). In simultaneous cytokinesis, meiosis II follows meiosis I without cell division. As a result, the four microspore nuclei occur within a common cytoplasm. Cell walls are deposited after meiosis II, forming a tetrad of spores usually in a tetrahedral arrangement. The spores are then released from the tetrad into the locule of the sporangium. This arrangement minimizes spatial cues about which spores are sisters and which are cousins. In successive cytokinesis, however, each division is followed by the formation of a cell wall, separating dyad nuclei after meiosis I and spore nuclei after meiosis II. Successive cytokinesis appears to provide more spatial cues about relatedness within tetrads and might therefore be more vulnerable to exploitation by driving alleles that employ such cues, although evidence on this point is lacking. Simultaneous cytokinesis appears to have been the ancestral mode in land plants with multiple or- 
igins of successive cytokinesis (Furness 2008; Nadot et al. 2008).

A modified form of sporogenesis occurs in the ovules of seed plants. In most cases, a single megasporocyte undergoes successive cytokinesis to form a tetrad of four megaspores. Three megaspores abort, and the survivor germinates within the ovule to produce a multicellular female gametophyte (Yadegari and Drews 2004). A similar suppression of three out of four products of meiosis occurs during metazoan oogenesis. At meiosis I, a primary oocyte divides asymmetrically to produce a secondary oocyte and first polar body, then the secondary oocyte divides asymmetrically to produce the functional egg and second polar body.

Abortion of all but one member of a tetrad also occurs during spermatogenesis in sciarid flies (Haig 1993b; Goday and Esteban 2001) and the formation of pseudomonad pollen in sedges (Kirpes et al. 1996) and epacrid heaths (Furness 2009), but asymmetric meiosis is a rarity in males rather than the rule in females. A plausible explanation is that greater benefits are obtained by megaspores (eggs) than by microspores (sperm) from resources scavenged from aborted tetrad mates.

Suppression of female meiotic products has been viewed as an adaptation of the diploid generation to ensure adequate food reserves for the developing embryo (Schuh and Ellenberg 2008). If spores had unit fitness in tetrads with symmetric meiosis, then each allele in a sporocyte would have obtained two units of fitness from a tetrad. Suppression of three out of four products of meiosis would have been favored as an adaptation of the diploid generation only if it more than quadrupled the fitness of the surviving spore, whereas it would be favored as an adaptation of the surviving spore if it more than doubled fitness (see "Winner Takes All").

Malik and Henikoff (2009) suggest that asymmetric female meiosis originated in a selfish strategy by which genes near centromeres benefited from killing other products of meiosis. My analysis suggests a minor modification: the expulsion of the first polar body was favored by genes close to centromeres, but the expulsion of the second polar body was favored by genes distant from centromeres. Even if asymmetric meiosis originated as a form of meiotic drive, the suppression of meiotic products may have subsequently been reinforced by selection on the diploid generation to prevent costly competition within tetrads. In other words, diploid fitness may have been optimized by choosing a winner before competition took place, even though higher fitness could have been obtained by provisioning all four spores in the absence of competition. This argument is analogous to the suggestion that a parent's optimal brood size may be smaller in the presence of sibling rivalry than in its absence (Godfray and Parker
1991). The origins of asymmetric female meiosis in animals and seed plants were ancient events, and these scenarios, although appealing, are unlikely to be directly testable.

\section{Drive at Female Meiosis I}

Asymmetric female meiosis creates opportunities for alleles that are able to bias their own segregation toward the functional product of meiosis (Zwick et al. 1999; Henikoff et al. 2001; Pardo-Manuel de Villena and Sapienza 2001).

Most cases of chromosomal nondisjunction in Drosophila and humans occur at female meiosis I. Zwick et al. (1999) proposed that this high rate of nondisjunction is a side effect of competition among centromeres to avoid inclusion in the first polar body. Human chromosome 16 is a strong candidate for such centromeric drive. A third of all human trisomies detected at miscarriage result from nondisjunction of chromosome 16 at maternal meiosis I (Hassold et al. 1991, 1995; Warburton et al. 1991; GarciaCruz et al. 2010). Moreover, trisomy 16 does not show the dramatic increase with maternal age of other trisomies (Risch et al. 1986), suggesting that nondisjunction of this chromosome has a different etiology from that of other chromosomes. A large block of pericentromeric heterochromatin is variable in size within human populations (Verma et al. 1978), and there is strong evidence of recent selection spanning this centromere (Williamson et al. 2007).

Centromeric drive requires symmetry breaking, either the creation or the exploitation of a difference between the two poles of the first meiotic spindle. The first meiotic spindle of female mice develops in the center of oocytes and lacks the astral microtubules that connect mitotic spindles to the cortex. At metaphase I, the spindle is pushed and pulled to the cortex by microfilaments. Movement is led by the spindle pole that happens to be nearer the cortex because of small eccentricities in spindle placement. Thus, when the spindle reaches the cortex, its axis is radial to the cell surface. Anaphase I immediately follows, and the outer group of chromosomes is pinched off in the first polar body (Verlhac et al. 2000; Maro and Verlhac 2002). A critical question is whether the orientation of centromeres with respect to the spindle poles is determined before or after determination of the direction of spindle migration. In the former case, a driving centromere must influence the direction of spindle movement, perhaps by interacting with microfilaments. In the latter case, a driving centromere must alter its orientation relative to the preexisting asymmetry of spindle poles.

By contrast, the first meiotic spindle of female dunnarts (marsupial "mice") forms in the periphery of the oocyte, with its axis parallel to the oocyte surface. After anaphase 
I, the spindle rotates $90^{\circ}$ to extrude the first polar body (Merry et al. 1995). Therefore, a dunnart centromere might drive by biasing the direction of spindle rotation or by biasing its own orientation relative to the extruded pole, if the latter is predetermined.

\section{Drive at Female Meiosis II}

The best characterized agent of preferential segregation at female meiosis II is abnormal chromosome 10 (Ab10) of maize. Ab10 also causes correlated drive of heterochromatic knobs on other chromosomes (Longley 1945; Dawe and Hiatt 2004). The differential segment of $A b 10$ and the heterochromatic knobs show precocious movement toward spindle poles at male meiosis I. Similar precocious movement during female meiosis, in combination with second-division segregation, is proposed to orient knobbearing chromatids toward the outer poles of spindles at meiosis II, causing the preferential inclusion of knobs in the functional chalazal megaspore (Rhoades and Dempsey 1966).

Two systems of segregation distortion have been described at female meiosis II in mice (HSR: Agulnik et al. 1993; Om: Wu et al. 2005). The second meiotic spindle forms in the cortex of mouse oocytes with its axis parallel to the oocyte surface. After anaphase II, the spindle rotates $90^{\circ}$ to allow expulsion of the second polar body (Maro and Verlhac 2002). Therefore, a possible mechanism of drive would be for a gene that has undergone seconddivision segregation to bias the direction of spindle rotation. By contrast, the second meiotic spindle of dunnarts is formed with its axis radial to the cell surface so that the second polar body is expelled without rotation of the spindle (Merry et al. 1995).

The differential segments of $A b 10$ in maize and HSR in mice both cause increased crossing-over between themselves and the centromere (Rhoades and Dempsey 1970; Gorlov et al. 1993). This effect should increase the frequency of second-division segregation and thus enhance the efficiency of drive. Heterochromatic knobs on other maize chromosomes are located at chromosomal locations that maximize drive at meiosis II (Buckler et al. 1999).

\section{Meiotic Drive and Recombination}

Competition within tetrads is a rich source of selective forces acting on recombination. In classical forms of drive, selection on unlinked modifiers favors increased recombination that dissipates linkage disequilibrium between distorter and responder loci and thereby reduces the intensity of drive (Haig and Grafen 1991). By contrast, in the nonclassical forms of drive discussed in this article, natural selection favors changes in recombination that en- hance drive. Selection acts on modifiers of crossing-over in these systems not because of effects on linkage disequilibrium but because crossing-over determines when alleles segregate. In principle, one could explain any observed pattern of crossing-over by choosing chromosomal locations of agents of drive and choosing particular parameter values in the models. Such flexibility makes the theory untestable unless these choices are constrained by data from actual systems of drive and from an understanding of differential vulnerabilities to drive in diverse taxa.

Several loci have now been identified that influence the activity and location of recombination hotspots (Paigen and Petkov 2010), including loci with allelic variants that have opposite effects on male and female recombination (Kong et al. 2008). Such studies suggest that recombination could respond rapidly to changes in selective forces. PRDM9 will illustrate the specificity and evolutionary lability of genetic effects on recombination. PRDM9 initiates recombination at hotspots in mice and humans (Baudat et al. 2010) and has undergone rapid evolution, especially in the zinc finger domains that directly interact with DNA (Oliver et al. 2009; Thomas et al. 2009). As a result, chimpanzee and human PRDM9 no longer recognize each other's hotspots (Myers et al. 2010). Selection on PRDM9 may differ depending on whether an allele is linked or unlinked to its allele-specific hotspots. A striking observation from comparative mapping is that PRDM9 appears to change location relative to flanking markers (PRDM9 is located between $\mathrm{CDH} 10$ and $\mathrm{CDH} 12$ on human chromosome 5 but between $C h d 1$ and $P d c d 2$ on mouse chromosome 17: NCBI build 37.1).

\section{Sex Differences in Recombination}

A difference between male and female meiosis in the frequency of recombination and localization of chiasmata (heterochiasmy) is commonly observed (Callan and Perry 1977; Hedrick 2007; Mank 2009). Lenormand (2003) concluded that sex-specific selection in the haploid phase is the most probable cause of these differences. Meiotic drive is a plausible candidate for sex-specific haploid selection because mechanisms of drive are usually specific to either male or female meiosis (Úbeda and Haig 2005). It should be emphasized that, in Lenormand's models, crossing-over is subject to selection because of its effects on linkage disequilibrium. Therefore, his models are particularly relevant to classical forms of meiotic drive that depend on linkage disequilibrium between distorter and responder loci (Haig and Grafen 1991). The nonclassical forms of drive discussed in this article are another possible source of sex-specific selection on recombination, but in this case, crossing-over is subject to selection because the location 
of chiasmata influences whether segregation occurs at meiosis I or II.

Greater overall recombination in females is a common finding (Hedrick 2007), but measures of overall recombination obscure sex differences of opposite sign in particular chromosome regions. For example, female recombination is greater than male recombination near centromeres, but male recombination exceeds female recombination near telomeres in humans (Lynn et al. 2004), mice (Shifman et al. 2006), dogs (Wong et al. 2010), and fish (Sakamoto et al. 2000; Singer et al. 2002). Such patterns could be interpreted as evidence for a significant role of drive at female meiosis II in shaping the distribution of chiasmata. By contrast, recombination is more frequent in male than in female marsupials (Zenger et al. 2002; Samollow et al. 2004), with chiasmata localized near telomeres in female dunnarts (Bennett et al. 1986).

Forms of drive that exploit the timing of segregation are expected to be more common in female meiosis than in male meiosis for two interrelated reasons. First, male meiosis provides fewer positional cues for distinguishing sisters from cousins. Second, nonrandom segregation confers no advantage if all four products of meiosis are equivalent (Pardo-Manuel de Villena and Sapienza 2001).

\section{Conclusions}

Competition may be particularly intense among the spores of a meiotic tetrad because relatedness to a random spore is one-third and relatedness to sisters or cousins approaches zero at some loci. Systems of meiotic drive are conjectured that use predictable patterns of segregation at meiosis I or meiosis II to exploit spores of low relatedness. Female meiosis is expected to be particularly susceptible to agents that preferentially segregate to the single functional product of a megasporocyte or oocyte.

Evolutionary theories of the function of crossing-over have emphasized effects on genetic recombination and linkage disequilibrium. Decay of linkage disequilibrium is not the only consequence of chiasmata, however. Most taxa require at least one crossover per bivalent to assure regular disjunction. This article proposes that selection on the timing of meiotic segregation can influence the localization of chiasmata and contribute to sex differences in recombination. Moreover, a fundamental trade-off exists such that patterns of crossing-over that are most conducive to drive at meiosis I are least conducive to drive at meiosis II, and vice versa. Empirical studies are needed to determine whether this source of selection plays a central or peripheral role in the localization of chiasmata.

\section{Acknowledgments}

This article has benefited from the comments of L. Chen, R. Trivers, and F. Úbeda. G. Sharman taught me that meiosis was a physical process taking place in four dimensions.

\section{Literature Cited}

Agulnik, S. I., I. D. Sabantsev, and A. O. Ruvinsky. 1993. Effect of sperm genotype on chromatid segregation in female mice heterozygous for aberrant chromosome. 1. Genetical Research 61:97100

Baudat, F., J. Buard, C. Grey, A. Fledel-Alon, C. Ober, M. Przeworski, G. Coop, and B. de Massy. 2010. PRDM9 is a major determinant of meiotic recombination hotspots in humans and mice. Science 327:836-840.

Bennett, J. H., D. L. Hayman, and R. M. Hope. 1986. Novel sex differences in linkage values and meiotic chromosome behaviour in a marsupial. Nature 323:59-60.

Buckler, E. S., T. L. Phelps-Durr, C. S. K. Buckler, R. K. Dawe, J. F. Doebley, and T. P. Holtsford. 1999. Meiotic drive of chromosomal knobs reshaped the maize genome. Genetics 153:415-426.

Burt, A., and R. Trivers. 2006. Genes in conflict. Harvard University Press, Cambridge, MA.

Callan, H. G., and P. E. Perry. 1977. Recombination in male and female meiocytes contrasted. Philosophical Transactions of the Royal Society B: Biological Sciences 277:227-233.

Charlesworth, B., and D. L. Hartl. 1978. Population dynamics of the segregation distorter polymorphism of Drosophila melanogaster. Genetics 89:171-192.

Dawe, R. K., and E. N. Hiatt. 2004. Plant neocentromeres: fast, focused, and driven. Chromosome Research 12:655-669.

Furness, C. A. 2008. Successive microsporogenesis in eudicots, with particular reference to Berberidaceae (Ranunculales). Plant Systematics and Evolution 273:211-223.

. 2009. Pollen evolution and development in Ericaceae, with particular reference to pseudomonads and variable pollen sterility in Styphelioideae. International Journal of Plant Sciences 170:476495.

Furness, C. A., P. J. Rudall, and F. B. Simpson. 2002. Evolution of microsporogenesis in angiosperms. International Journal of Plant Sciences 163:235-260.

Garcia-Cruz, R., A. Casanovas, M. Brieño-Enríquez, P. Robles, I. Roig, A. Pujol, L. Cabero, M. Durban, and M. G. Caldés. 2010. Cytogenetic analyses of human oocytes provide new data on nondisjunction mechanisms and the origin of trisomy 16. Human Reproduction 25:179-191.

Gardner, A., and S. A. West. 2010. Greenbeards. Evolution 64:2538.

Goday, C., and M. R. Esteban. 2001. Chromosome elimination in sciarid flies. BioEssays 23:242-250.

Godfray, H. C. J., and G. A. Parker. 1991. Clutch size, fecundity and parent-offspring conflict. Philosophical Transactions of the Royal Society B: Biological Sciences 332:67-79.

Gorlov, I. P., T. Y. Ladygina, and P. M. Borodin. 1993. Chiasma distribution in the first bivalent of mice carrying a double insertion of homogeneously-staining regions in homo- and heterozygous states. Heredity 70:642-647.

Haig, D. 1986. Conflicts among megaspores. Journal of Theoretical Biology 123:471-480. 
1993a. Alternatives to meiosis: the unusual genetics of red algae, microsporidia, and others. Journal of Theoretical Biology $163: 15-31$.

. 1993b. The evolution of unusual chromosomal systems in sciarid flies: intragenomic conflict and the sex ratio. Journal of Evolutionary Biology 6:249-261.

. 1997. The social gene. Pages 284-304 in J. R. Krebs and N. B. Davies, eds. Behavioural ecology. 4th ed. Blackwell Scientific, Oxford.

Haig, D., and A. Grafen. 1991. Genetic scrambling as a defence against meiotic drive. Journal of Theoretical Biology 153:531-558.

Hassold, T. J., D. Pettay, S. B. Freeman, M. Grantham, and N. Takaesu. 1991. Molecular studies of non-disjunction in trisomy 16. Journal of Medical Genetics 28:159-162.

Hassold, T. J., M. Merrill, K. Adkins, S. Freeman, and S. Sherman. 1995. Recombination and maternal age-dependent nondisjunction: molecular studies of trisomy 16. American Journal of Human Genetics 57:867-874.

Hedrick, P. W. 2007. Sex: differences in mutation, recombination, selection, gene flow, and genetic drift. Evolution 61:2750-2771.

Henikoff, S., K. Ahmad, and H. S. Malik. 2001. The centromere paradox: stable inheritance with rapidly evolving DNA. Science 293:1098-1102.

Kirpes, C. C., L. G. Clark, and N. R. Lersten. 1996. Systematic significance of pollen arrangement in microsporangia of Poaceae and Cyperaceae: review and observations on representative taxa. American Journal of Botany 83:1609-1622.

Kong, A., G. Thorleifsson, H. Stefansson, G. Masson, A. Helgason, D. F. Gudbjartsson, G. M. Jonsdottir, et al. 2008. Sequence variants in the RNF212 gene associate with genome-wide recombination rate. Science 319:1398-1401.

Lenormand, T. 2003. The evolution of sex dimorphism in recombination. Genetics 163:811-822.

Longley, A. E. 1945. Abnormal segregation during megasporogenesis in maize. Genetics 30:100-113.

Lynn, A., T. Ashley, and T. Hassold. 2004. Variation in human meiotic recombination. Annual Review of Genomics and Human Genetics 5:317-349.

Lyttle, T. W. 1991. Segregation distorters. Annual Review of Genetics 25:511-557.

Malik, H. S., and S. Henikoff. 2009. Major evolutionary transitions in centromere complexity. Cell 138:1067-1082.

Mank, J. E. 2009. The evolution of heterochiasmy: the role of sexual selection and sperm competition in determining sex-specific recombination rates in eutherian mammals. Genetics Research 91: 355-363.

Maro, B., and M.-H. Verlhac. 2002. Polar body formation: new rules for asymmetric divisions. Nature Cell Biology 4:E281-E283.

Mather, K. 1935. Reductional and equational separation of the chromosomes in bivalents and multivalents. Journal of Genetics 30: $53-78$.

McCormick, S. 2004. Control of male gametophyte development. Plant Cell 16:S142-S153.

Merry, N. E., M. H. Johnson, C. A. Gehring, and L. Selwood. 1995. Cytoskeletal organization in the oocyte, zygote, and early cleaving embryo of the stripe-faced dunnart (Sminthopsis macroura). Molecular Reproduction and Development 41:212-224.

Myers, S., R. Bowden, A. Tumian, R. E. Bontrop, C. Freeman, T. S. MacFie, G. McVean, and P. Donnelly. 2010. Drive against hotspot motifs in primates implicates the PRDM9 gene in meiotic recombination. Science 327:876-879.

Nadot, S., C. A. Furness, J. Sannier, L. Penet, B. Triki-Teurtroy, and A. Ressayre. 2008. Phylogenetic comparative analysis of microsporogenesis in angiosperms with a focus on monocots. American Journal of Botany 95:1426-1436.

Oliver, P. L., L. Goodtsadt, J. J. Bayes, Z. Birtle, K. C. Roach, N. Phadnis, S. A. Beatson, G. Lunter, H. S. Malik, and C. P. Ponting. 2009. Accelerated evolution of the Prdm9 speciation gene across diverse metazoan taxa. PLoS Genetics 5:e1000753.

Paigen, K., and P. M. Petkov. 2010. Mammalian recombination hot spots: properties, control and evolution. Nature Reviews Genetics 11:221-233.

Pardo-Manuel de Villena, F., and C. Sapienza. 2001. Nonrandom segregation during meiosis: the unfairness of females. Mammalian Genome 12:332-339.

Raju, N. B., and D. D. Perkins. 1994. Diverse programs of ascus development in pseudohomothallic species of Neurospora, Gelasinospora, and Podospora. Developmental Genetics 15:104-118.

Rhoades, M. M., and E. Dempsey. 1966. The effect of abnormal chromosome 10 on preferential segregation and crossing over in maize. Genetics 53:989-1020.

- 1970. Crossing over and preferential segregation in chromosome 10 induced by abnormal chromosome 10. Maize Genetics Cooperation News Letter 44:57-58.

Risch, N., Z. Stein, J. Kline, and D. Warburton. 1986. The relationship between maternal age and chromosome size in autosomal trisomy. American Journal of Human Genetics 39:68-78.

Sakamoto, T., R. G. Danzmann, K. Gharbi, P. Howard, A. Ozaki, S. K. Khoo, R. A. Woram, et al. 2000. A microsatellite linkage map of rainbow trout (Oncorhynchus mykiss) characterized by large sexspecific differences in recombination rates. Genetics 155:13311345.

Samollow, P. B., C. M. Kammerer, S. M. Mahaney, J. L. Schneider, S. J. Westenberger, J. L. VandeBerg, and E. S. Robinson. 2004. First-generation linkage map of the gray, short-tailed opossum, Monodelphis domestica, reveals genome-wide reduction in female recombination rates. Genetics 166:307-329.

Schuh, M., and J. Ellenberg. 2008. A new model for asymmetric spindle positioning in mouse oocytes. Current Biology 18:19861992.

Shifman, S., J. T. Bell, R. R. Copley, M. S. Taylor, R. W. Williams, R. Mott, and J. Flint. 2006. A high-resolution single nucleotide polymorphism genetic map of the mouse genome. PLoS Biology 4:e395.

Singer, A., H. Perlman, Y. Yan, C. Walker, G. Corley-Smith, B. Brandhorst, and J. Postlethwait. 2002. Sex-specific recombination rates in zebrafish (Danio rerio). Genetics 160:649-657.

Thomas, J. H., R. O. Emerson, and J. Shendure. 2009. Extraordinary molecular evolution in the PRDM9 fertility gene. PLoS One 4: e8505.

Turner, B. C., and D. D. Perkins. 1991. Meiotic drive in Neurospora and other fungi. American Naturalist 137:416-429.

Úbeda, F., and D. Haig. 2005. On the evolutionary stability of Mendelian segregation. Genetics 170:1345-1357.

Verlhac, M. H., C. Lefebvre, P. Guillard, P. Rassinier, and B. Maro. 2000. Asymmetric division in mouse oocytes: with or without Mos. Current Biology 10:1303-1306.

Verma, R. S., H. Dosik, and H. A. Lubs. 1978. Size and pericentric inversion heteromorphisms of secondary constriction regions (h) 
of chromosomes 1,9 , and 16 as detected by CBG technique in Caucasians. American Journal of Medical Genetics 3:331-339.

Warburton, D., J. Byrne, and N. Canki. 1991. Chromosome anomalies and prenatal development: an atlas. Oxford University Press, New York.

Williamson, S. H., M. J. Hubisz, A. G. Clark, B. A. Payseur, C. D. Bustamante, and R. Nielsen. 2007. Localizing recent adaptive evolution in the human genome. PLoS Genetics 3:e90.

Wong, A. K., A. L. Ruhe, B. L. Dumont, K. R. Robertson, G. Guerrero, S. M. Shull, J. S. Ziegle, et al. 2010. A comprehensive linkage map of the dog genome. Genetics 184:595-605.

Wu, G., L. Hao, Z. Han, S. Gao, K. E. Latham, F. Pardo-Manuel de Villena, and C. Sapienza. 2005. Maternal transmission ratio distortion at the mouse $\mathrm{Om}$ locus results from meiotic drive at the second meiotic division. Genetics 170:327-334.
Yadegari, R., and G. N. Drews. 2004. Female gametophyte development. Plant Cell 16:S133-S141.

Zenger, K. R., L. M. McKenzie, and D. W. Cooper. 2002. The first comprehensive genetic linkage map of a marsupial: the tammar wallaby (Macropus eugenii). Genetics 162:321-330.

Zwick, M. E., J. L. Salstrom, and C. H. Langley. 1999. Genetic variation in rates of nondisjunction: association of two naturally occurring polymorphisms in the chromokinesin nod with increased rates of nondisjunction in Drosophila melanogaster. Genetics 152: $1605-1614$
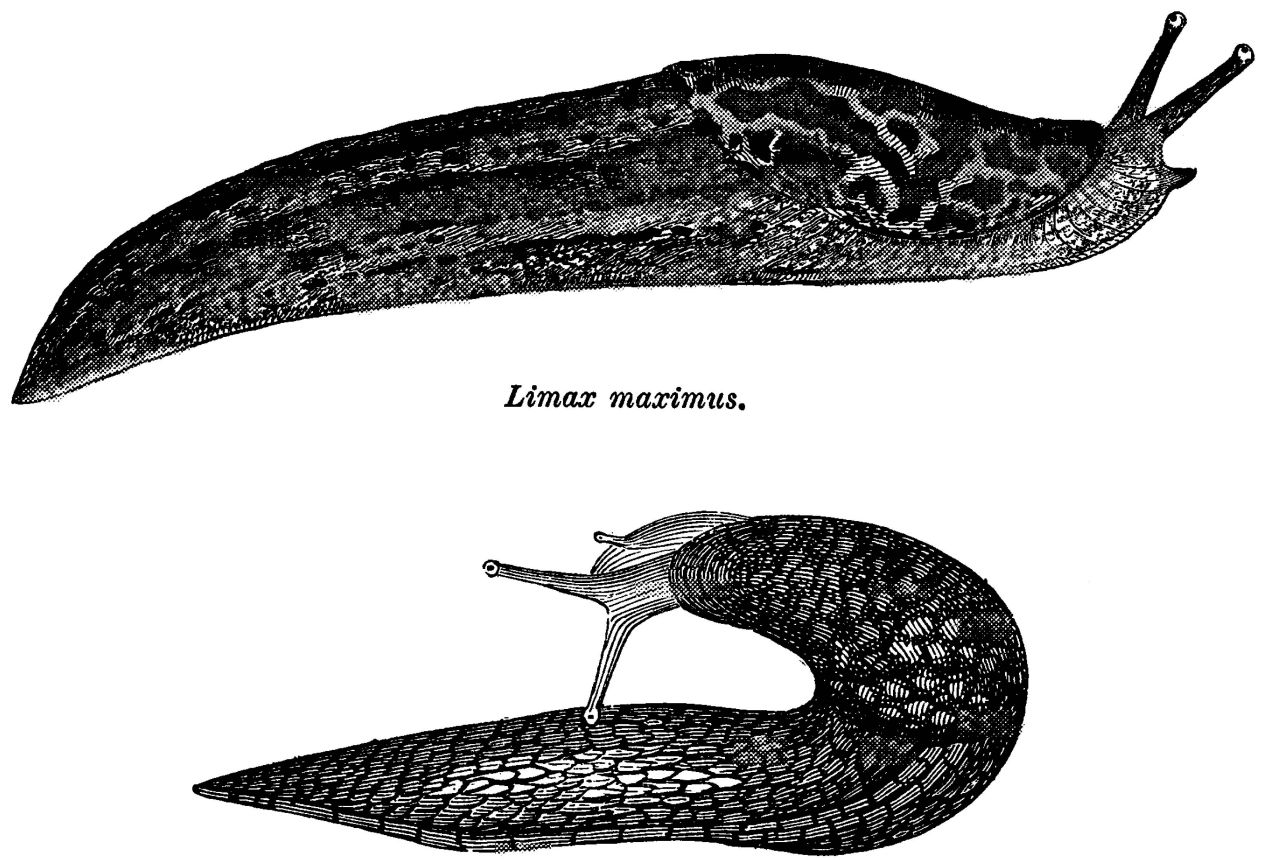

Limax flavus.

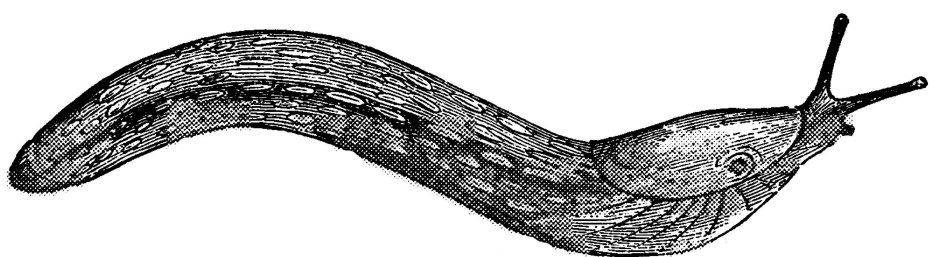

Arion fuscus.

"Limax maximus is the largest of our cellar slugs. It seems to be a more recent importation than the other species, having first been noticed in Philadelphia in 1867. ... Limax flavus has been noticed for more than forty years in the cities of our Atlantic coast. ... Arion fuscus is not properly a cellar snail, but is found with the preceding species around kitchens and gardens." From "The Mollusks of Our Cellars" by W. G. Binney (American Naturalist, 1870, 4:166-171). 